\title{
Axiomatic Design of Automobile Suspension and Steering Systems: Proposal for a novel six-bar suspension
}

\author{
Hrishikesh V. Deo, Nam P. Suh \\ Massachusetts Institute of Technology
}

Copyright (C) 2003 SAE International

\begin{abstract}
The existing vehicle designs exhibit a high level of coupling. For instance the coupling in the suspension and steering systems manifests itself through the change in wheel alignment parameters (WAP) due to suspension travel. This change in the WAP causes directional instability and tire-wear. The approach of the industry to solve this problem has been twofold. The first approach has been optimization of suspension link lengths to reduce the change in WAP to zero. Since this is not possible with the existing architecture, the solution used is the optimization of the spring stiffness $\mathrm{K}$ to get a compromise solution for comfort (which requires significant suspension travel and hence a soft spring) and directional stability (which demands least possible change in wheel alignment parameters and hence a stiff spring).
\end{abstract}

This paper presents an axiomatic design solution to this problem and an attempt to remove the coupling in the steering and suspension systems by making the WAP independent of suspension travel. The four-bar linkages used in the existing independent suspension systems are incapable of satisfying their FRs and cause coupling at a higher level. The proposed solution uses a six-bar Watt-I linkage suspension, which removes the coupling. It offers other advantages like the hardening characteristics for the suspension. A new steering system conformal to the new suspension system has been proposed.

FR/DP decomposition of the vehicle systems is presented. This indicates other couplings and DP redundancies in the vehicle system and also provides the framework for design of novel vehicles.

\section{INTRODUCTION}

\section{INTRODUCTION TO AXIOMATIC DESIGN [1, 2]}

Axiomatic Design is a structured design method created to improve design activities by establishing criteria on which potential designs may be evaluated and by developing tools for implementing these criteria. Axiomatic design discusses the existence of four domains in the design world- customer, functional, physical and process domains. Customer attributes $\{C A s\}$, functional requirements $\{F R s\}$, design parameters $\{D P s\}$, and process variables $\{P V s\}$ are the characteristic vectors of these domains. Design of products involves mapping from the functional domain to the physical domain and design of processes involves mapping from the physical domain to the process domain.

The axiomatic design process is centered on the satisfaction of FRs, which are defined as the minimum set of independent requirements that completely characterize the functional need of the product. Given a minimum set of independent FRs, the designer conceives a physical embodiment or a design containing a set of DPs, which are key physical variables in the physical domain that characterize the design that satisfies the specified FRs. The design and the choice of DPs are guided by the two design axioms.

- Axiom 1: Independence Axiom- Maintain the independence of all functional requirements.

- Axiom 2: Information Axiom- Minimize the information content of the design.

The design matrix (DM) is used to note the effect of DPs on FRs as follows:

$$
\left\{\begin{array}{l}
F R 1 \\
F R 2
\end{array}\right\}=\left[\begin{array}{ll}
A_{11} & O \\
A_{21} & A_{22}
\end{array}\right]\left\{\begin{array}{l}
D P 1 \\
D P 2
\end{array}\right\} \ldots(1)
$$

where $A_{11}$ denotes the effect of DP 1 on FR $1, A_{21}$ denotes the effect of DP 1 on FR 2, etc. To satisfy the Independence Axiom, the DM must be must be either diagonal or triangular. In an uncoupled design, the DM is diagonal and each of the FRs can be satisfied independently by adjusting one DP. In a decoupled design, the matrix is triangular and the independence of FRs can be guaranteed only if the DPs are determined in a proper sequence. In the case shown, we need to set the DPs in the order: DP 1 followed by DP 2. A full design matrix leads to a coupled design and the satisfaction of FRs becomes difficult. 
The Information Axiom guides the designer to maximize the probability of satisfaction of the FRs. It becomes increasingly difficult to satisfy FRs when FRs are coupled by the chosen DPs. This is because the allowable tolerance for DPs decreases with the increase in the number of FRs and the number of off-diagonal elements in the design matrix.

\section{WHEEL ALIGNEMENT PARAMETERS $[3,5,8]$}

Orientation of the wheels and steering axes with respect to the vehicle frame and with respect to the terrain changes due to suspension travel. Figure 1 shows the wheel alignment parameters which describe the orientation of the wheel and the wheel axis. Excess camber causes tire wear and camber spread causes directional instability. Caster spread causes directional instability. Toe change due to suspension travel causes Bump Steer and excess toe causes tire-wear. Because of these factors vehicles exhibit tire-wear and directional instability due to suspension travel under conditions of overload, offset load or road undulations.



Figure 1 Wheel Alignment Parameters FRDP DECOMPOSITION OF VEHICLE SYSTEM

Table 1 shows the top level FR/DP decomposition for the vehicle system and the design matrix (DM) is shown in Equation 2. The DM indicates two sets of couplingscoupling between FR 13 and FR 15 and coupling between FR 14 and FR 15 . The effect of DP 15 on FR 13 is small (as indicated by $x$ in the DM in Equation 2) and the design works in spite of this coupling due to the presence of a feedback control system- the driver. Identification and removal of the coupling between FR 14 and FR 15 is the subject matter of this paper. These two FRs have been decomposed further to understand this coupling better.

Table 2 shows the decomposition of FR 14: Hold passengers. As indicated in the decomposition, the passenger compartment must provide safety, comfort and pleasure to the passenger. FR/DP decomposition for
FR 142: Ensure comfortable ride is shown in Table 3. The corresponding DM is given in Equation 3.

Table 1: Top level FR/DP decomposition of vehicle system

\begin{tabular}{|l|l|l|}
\hline & $\begin{array}{l}\text { Functional } \\
\text { Requirements }\end{array}$ & Design Parameters \\
\hline Parent & $\begin{array}{l}\text { Need for } \\
\text { transportation }\end{array}$ & Vehicle system \\
\hline 11 & $\begin{array}{l}\text { Allow low resistance } \\
\text { motion }\end{array}$ & $\begin{array}{l}\text { Rolling motion } \\
\text { (Wheels) }\end{array}$ \\
\hline 12 & Hold cargo & Cargo space \\
\hline 13 & Control speed & $\begin{array}{l}\text { Wheel rotation } \\
\text { speed }\end{array}$ \\
\hline 14 & Hold passengers & Passenger space \\
\hline 15 & Control direction & Turning torque \\
\hline 16 & Attractive appearance & Exterior Bodywork \\
\hline
\end{tabular}

$\left\{\begin{array}{l}F R 11 \\ F R 12 \\ F R 13 \\ F R 14 \\ F R 15 \\ F R 16\end{array}\right\}=\left[\begin{array}{llllll}X & O & O & O & O & O \\ X & X & O & O & O & O \\ X & O & X & O & x & O \\ X & O & X & X & X & O \\ X & O & X & X & X & O \\ X & X & O & X & X & X\end{array}\right]\left\{\begin{array}{l}D P 11 \\ D P 12 \\ D P 13 \\ D P 14 \\ D P 15 \\ D P 16\end{array}\right\} \ldots$

Table 2 : FR/DP decomposition of FR14 (Hold passengers)

\begin{tabular}{|l|l|l|}
\hline & $\begin{array}{l}\text { Functional } \\
\text { Requirements }\end{array}$ & Design Parameters \\
\hline Parent & Hold passenger & $\begin{array}{l}\text { Passenger } \\
\text { compartment }\end{array}$ \\
\hline 141 & Provide crash protection & Impact strength \\
\hline 142 & Ensure comfortable ride & $\begin{array}{l}\text { Suspension } \\
\text { dynamics }\end{array}$ \\
\hline 143 & $\begin{array}{l}\text { Provide pleasing } \\
\text { environment }\end{array}$ & Interior design \\
\hline
\end{tabular}

Table 3 : FR/DP decomposition of FR142

\begin{tabular}{|l|l|l|}
\hline & $\begin{array}{l}\text { Functional } \\
\text { Requirements }\end{array}$ & Design Parameters \\
\hline Parent & $\begin{array}{l}\text { Ensure comfortable } \\
\text { ride }\end{array}$ & $\begin{array}{l}\text { Suspension } \\
\text { dynamics }\end{array}$ \\
\hline 1421 & $\begin{array}{l}\text { Limit maximum relative } \\
\text { motion }\end{array}$ & Spring rate \\
\hline 1422 & Dissipate energy & Damping coefficient \\
\hline 1423 & Set equilibrium position & Spring initial length \\
\hline
\end{tabular}


Table 4 shows the decomposition of FR 15 (Control direction) and the corresponding design matrix is given in Equation 4.

Table 4 : FR/DP decomposition of FR15

\begin{tabular}{|l|l|l|}
\hline & $\begin{array}{l}\text { Functional } \\
\text { Requirements }\end{array}$ & Design Parameters \\
\hline Parent & Control direction & Turning torque \\
\hline 151 & $\begin{array}{l}\text { Maintain wheel } \\
\text { alignment }\end{array}$ & $\begin{array}{l}\text { Suspension } \\
\text { kinematics }\end{array}$ \\
\hline 152 & $\begin{array}{l}\text { Maintain tire-road } \\
\text { contact }\end{array}$ & Suspension travel \\
\hline 153 & Adjust desired torque & Wheel angle \\
\hline
\end{tabular}

$$
\left\{\begin{array}{l}
F R 151 \\
F R 152 \\
F R 153
\end{array}\right\}=\left[\begin{array}{lll}
X & O & O \\
X & X & O \\
X & O & X
\end{array}\right]\left\{\begin{array}{l}
D P 151 \\
D P 152 \\
D P 153
\end{array}\right\} \ldots(4)
$$

In several complex systems, a coupled DM at the highest level may be decoupled by system-wide rearrangement of the DPs and FRs [4]. It was observed that it is difficult to come up with a design which would remove the coupling between FR 14 and FR 15 at the highest level. But it is possible to have a decoupled system after decomposition. To illustrate this, FR 142 and FR 15 are decomposed together and the corresponding DM presented in Equation 5.

$\left\{\begin{array}{c}F R 151 \\ F R 152 \\ F R 1421 \\ F R 1422 \\ F R 1423 \\ F R 153\end{array}\right\}=\left[\begin{array}{cccccc}X & X_{1} & X_{3} & O & O & O \\ X & X & O & O & O & O \\ X & O & X & O & O & O \\ X & O & X & X & O & O \\ X & O & X & O & X & O \\ X & X_{2} & X_{4} & O & O & X\end{array}\right]\left\{\begin{array}{c}D P 151 \\ D P 152 \\ D P 1421 \\ D P 1422 \\ D P 1423 \\ D P 153\end{array}\right\} \ldots(5)$

\section{EXISTING DESIGNS: IDENTIFICATION OF COUPLING}

The elements $X_{1}, X_{2}$, and $X_{3}$ in the DM in Equation 5, indicate the coupling. $X_{1}$ indicates that suspension travel causes the WAP to change and this causes unwanted turning torque changes, as indicated by $\mathrm{X}_{2}$. The extent to which the WAP change and hence the magnitude of the unwanted turning torque change depends on the spring stiffness as indicated by the elements $X_{3}$ and $X_{4}$. Note that $X_{2}$ being non-zero does not make the DM coupled. But DP 152 (Suspension travel) is a dynamic DP and it affects FR 153 (Adjust desired torque). Hence, to satisfy FR 153, we would require real-time adjustment of DP 153 (Wheel angle). To avoid this, we need a design that is uncoupled with respect to the dynamic design parameter DP $152\left(\mathrm{X}_{1}=0, \mathrm{X}_{2}=0\right)$.

These elements can be made zero and coupling can be removed by making the WAP independent of suspension travel. This section examines the change in WAP due to suspension travel in the existing designs, lists the problems due to this coupling and explores the possibility of removing this coupling by making the WAP independent of suspension travel.

\section{EXISTING SUSPENSION SYSTEMS: FOUR-BAR LINKAGES}

All existing front-wheel independent suspension systems are variations of the four-bar mechanism. For instance, the parallel arm suspension, the short long arm (SLA) suspension and the McPherson strut suspension can be kinematically represented as shown in Figure 2.

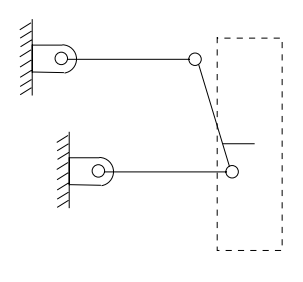

Parallel Arm Suspension

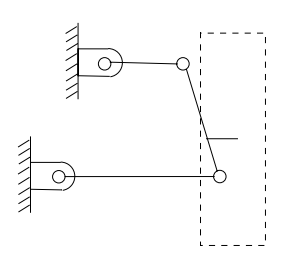

LSA Suspension

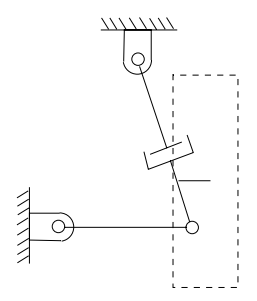

McPherson Strut

\section{Figure 2: Kinematic representation of independent suspensions}

The FRs that DP 151: Suspension Kinematics (Four-bar linkage) is expected to satisfy are given in the form of FR/DP decomposition in Table 5. We will only look into the following three important FRs for simplifying the analysis in this paper: Provide relative Z-motion, Avoid track changes $(\Delta y=0)$ and Avoid camber and caster changes $(\Delta \emptyset=0)$. Here $\Delta y$ indicates tire scrub and $\Delta \varnothing$ indicates camber change. The other FRs are uncoupled and can be easily satisfied.

Table 5 : FR/DP decomposition of FR151 (Maintain wheel alignment)

\begin{tabular}{|l|l|l|}
\hline & $\begin{array}{l}\text { Functional } \\
\text { Requirements }\end{array}$ & Design Parameters \\
\hline Parent & $\begin{array}{l}\text { Maintain wheel } \\
\text { alignment }\end{array}$ & $\begin{array}{l}\text { Suspension } \\
\text { kinematics }\end{array}$ \\
\hline 1511 & Permit relative Z-motion & $\begin{array}{l}\text { Single degree of } \\
\text { freedom system }\end{array}$ \\
\hline 1512 & $\begin{array}{l}\text { Avoid track changes } \\
(\Delta y=0)\end{array}$ & $\begin{array}{l}\text { Effective swing } \\
\text { axle radius }\end{array}$ \\
\hline 1513 & $\begin{array}{l}\text { Avoid camber and } \\
\text { caster changes }(\Delta \emptyset=0)\end{array}$ & $\begin{array}{l}\text { Equal motion of } \\
\text { steering axis joints }\end{array}$ \\
\hline
\end{tabular}

Analysis of the parallel arm suspension shows that it is capable of providing relative $\mathrm{Z}$ motion by change of angle $\theta$ as shown in Figure 4 and can maintain $\Delta \varnothing=0$ as both joints of the steering axis have equal vertical motion during suspension travel. But the parallel-arm suspension is incapable of satisfying FR 1512: $\Delta y=0$ during suspension travel. This causes tire-scrub due to suspension travel as illustrated in Figure 4.

In the SLA suspension, we can achieve $\Delta y=0$ (no tire scrub) through assignment of appropriate values to the 
link lengths, but this doesn't allow $\Delta \varnothing=0$ during suspension travel. This causes camber change and caster change due to suspension travel [5]. A compromise solution for $\Delta y$ and $\Delta \varnothing$ can be obtained through optimization of the link lengths and joint positions, but we cannot satisfy all three FRs simultaneously using a four bar linkage. Both $\Delta \mathrm{y}$ and $\Delta \varnothing$ can be reduced by increasing the link lengths, but this is limited by the constraints of cost, packaging and unsprung weight of the vehicle

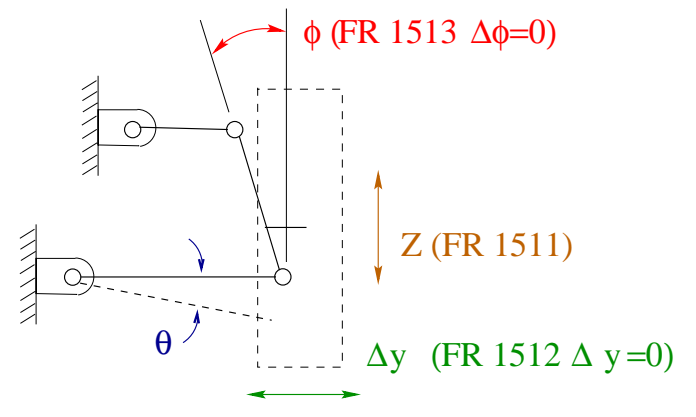

Figure 3: Representation of the FRs of the suspension system



\section{Figure 4: Kinematic representation of parallel-arm suspension showing tire-scrub}

The McPherson strut suspension, also a four-bar linkage with one prismatic joint, is incapable of satisfying the three FRs simultaneously as well. It exhibits tire-scrub as well as WAP changes due to suspension travel.

This implies that in the existing designs, suspension travel affects the WAP. This makes the system coupled as indicated by the elements $X_{1}, X_{2}$ and $X_{3}$ in Equation 5 . This coupling leads to several problems. The changes in camber angle and toe due to excess suspension travel under overload causes unnecessary tire-wear. This could be a serious issue in trucks as the WAP could change significantly from unloaded to fully loaded condition. Under offset load, different suspension travel for the wheels could cause camber spread, caster spread or toe spread leading to directional instability or Drift/pull of the vehicle. Toe change due to suspension travel causes Bump Steer due to road undulations. Toe change due to suspension travel is also a possible source for the Nibble problem, in which the high frequency road noises are transmitted back to the steering wheel.

\section{Manifestation of coupling in existing systems}

Very often in coupled designs, when one DP affects two or more FRs, these FRs require the DP to have different values. This leads to a trade-off between the conflicting FRs and the designer has to resort to optimization of the DPs to achieve the best compromise solution. The coupling in the automobile suspension and steering system is manifested by the following trade-offs to achieve compromise solutions:

1. Compromise between $\Delta \varnothing=0$ and $\Delta y=0$ through optimization of link lengths.

2. Compromise between control and comfort through optimization of spring stiffness $\mathrm{K}$. Control demands a stiff suspension, whereas comfortable ride demands a soft suspension.

Since the existing designs cannot make the WAP independent of suspension travel, optimization of the spring stiffness has been the approach of the industry to get a compromise solution for FRs of comfort and control. The axiomatic design approach points out the coupling between the FRs and indicates the need for developing a new uncoupled solution so that we do not have to live with the compromise solutions indicated above. Axiomatic design theory suggests the need for a new design that can satisfy both $\Delta \varnothing=0$ and $\Delta y=0$ simultaneously. Such a design would make the WAP independent of suspension travel and spring stiffness. Hence the control of the vehicle will improve. Spring stiffness can be designed only from comfort considerations and this will also improve passenger comfort.

\section{PROPOSED NEW DESIGN}

This section discusses the new suspension and steering system proposed to remove the identified coupling.

\section{PROPOSED DESIGN OF A SUSPENSION SYSTEM}

Analysis in the previous section indicated that the fourbar linkage is incapable of satisfying all three FRs (provide suspension travel, maintain $\Delta \emptyset=0$ and maintain $\Delta y=0)$ simultaneously. This leads to coupling at a higher level. The DM indicates that the coupling can be removed by a change of DP 151: Suspension kinematics (Four-bar mechanism). A decision was made to change to DP 151: Suspension kinematics (Single degree of freedom system). Stating this as the DP presents several single degree of freedom systems as options for the hardware of the suspension kinematics, namely- a single revolute or prismatic joint, six-bar linkage and so on, apart from the four-bar linkage.

A prismatic joint is used in two wheeler suspensions, but there are issues involved in incorporating it in an automobile suspension. Revolute joint is used in the swing-axle suspension and it is capable of meeting only one FR out of the three FRs simultaneously. In a swing- 
axle suspension, both camber and track changes during suspension travel (neither $\Delta y=0$, nor $\Delta \varnothing=0$ ).

The five possible inversions of a six-bar linkage using all revolute joints are shown in Figure 5 . Investigation revealed that the Stephenson chains and the Watt-II chain are incapable of satisfying the three FRs simultaneously. Hence we proceed with the dimensional synthesis of the Watt-I linkage to see if it can satisfy the three FRs simultaneously.
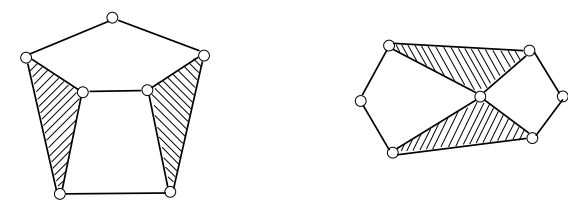

Stephenson Linkage

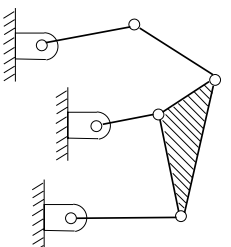

S III

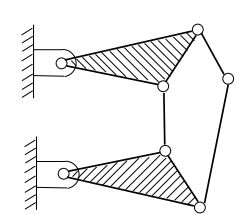

S I
Watt Linkage

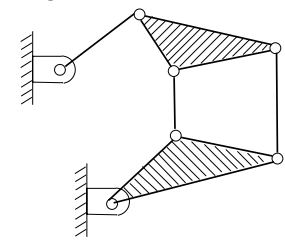

S II

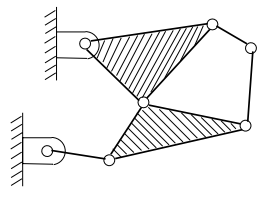

W I

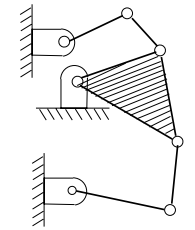

W II
Figure 5: Different possible six-bar mechanisms

\section{DIMENSIONAL SYNTHESIS OF WATT-I LINKAGE FOR THE SUSPENSION}

Complex number representation is very convenient to model linkage members and their motions, and is very commonly used in kinematic analysis and synthesis [6]. A great majority of planar linkages can be thought of as combinations of vector pairs called dyads. For instance the four-bar linkage in Figure 8 can be perceived of as two dyads: the left side of the linkage represented as a vector pair $\mathbf{W}$ and $\mathbf{Z}$, and the right side represented by the dyad $\mathbf{W}^{*}$ and $\mathbf{Z}^{*}$. The path point $\mathrm{P}$ of the coupler moves along a path from position $P_{1}$ to $P_{j}$ defined in an arbitrary complex coordinate system by $\mathbf{R}_{1}$ and $\mathbf{R}_{j}$.

Suppose we specify two positions for an unknown dyad by prescribing the values of $\mathbf{R}_{1}, \mathbf{R}_{\mathrm{j}} \alpha_{\mathrm{i}}$ and $\beta_{\mathrm{j}}$. To find the unknown starting position vectors of the dyad $\mathbf{W}$ and $\mathbf{Z}$, a loop-closure equation may be derived by summing the vectors clockwise around the loop containing $W \exp \left\{i \alpha_{j}\right\}$, $\mathbf{Z} \exp \{i \beta j\}, \mathbf{W}$ and $\mathbf{Z}$ :

$$
\mathbf{W}\left(e^{i \boldsymbol{\alpha}_{j}}-1\right)+\mathbf{Z}\left(e^{i \boldsymbol{\beta}_{j}}-1\right)=\boldsymbol{\delta}_{j} \ldots
$$

where $\boldsymbol{\delta}_{j}=\mathbf{R}_{j}-\mathbf{R}_{1}$ s the displacement vector along the prescribed trajectory from $P_{1}$ to $P_{j}$. This equation is called the standard-form equation and is simply the vector sum around the loop containing the first and the $\mathrm{j}^{\text {th }}$ positions of the dyad forming the left side of the four-bar linkage. This synthesis technique is well developed for synthesis of four-bar mechanisms for different tasks: Path generation, motion generation and function generation [6]. It involves expressing the desired motions and/or angular displacements of the input, output or coupler links (depending on the application) in the standard-form equation and solving these equations to get the desired link lengths. This enables us to get an analytical solution to the synthesis problem. The next section discusses the application of this technique for the synthesis of the suspension system from the Watt-I linkage

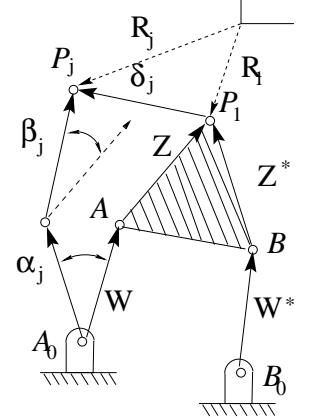

Figure 6 : Notation for the dyad

\section{APPLICATION TO SUSPENSION DESIGN}

Table 6 shows the FRs and the constraints that DP 151: Suspension kinematics is supposed to satisfy. Figure 7 shows the application of the dyad technique for the synthesis of the suspension system using the six-bar Watt-I linkage. Here $\boldsymbol{\delta}_{j}$ indicates the displacement of the steering axis joints $A$ and $B$. Since $\boldsymbol{\delta}_{j}$ are same for $A$ and $\mathrm{B}, \boldsymbol{\delta}_{j}$ is also the displacement of the steering axis as a whole. $\mathbf{Z}_{1}$ through $\mathbf{Z}_{6}$ are vectors which characterize the link lengths and the existing state of the mechanism. The angular rotations of the links 1 to 4 are given by $\alpha_{i}, \beta_{i}, Y_{i}$ and $\theta_{i}$ respectively as shown in Figure 7 .

Table 6 : FR/DP decomposition of FR 151 (Maintain wheel alignment)

\begin{tabular}{|l|l|l|}
\hline & $\begin{array}{l}\text { Functional } \\
\text { Requirements }\end{array}$ & Design Parameters \\
\hline Parent & $\begin{array}{l}\text { Maintain wheel } \\
\text { alignment }\end{array}$ & $\begin{array}{l}\text { Suspension } \\
\text { kinematics }\end{array}$ \\
\hline 1511 & Permit relative Z-motion & $\begin{array}{l}\text { Single degree of } \\
\text { freedom system }\end{array}$ \\
\hline 1512 & $\begin{array}{l}\text { Avoid track changes } \\
(\Delta \mathrm{y}=0)\end{array}$ & $\operatorname{Im}\left(\boldsymbol{\delta}_{j}\right)=0$ \\
\hline 1513 & $\begin{array}{l}\text { Avoid camber and } \\
\text { caster changes }(\Delta \emptyset=0)\end{array}$ & $\begin{array}{l}\text { Equal motion of } \\
\text { steering axis joints }\end{array}$ \\
\hline 1514 & $\begin{array}{l}\text { Hardening } \\
\text { characteristics }\end{array}$ & $\alpha_{i}$ \\
\hline
\end{tabular}




$$
\left\{\begin{array}{l}
F R 1511 \\
F R 1512 \\
F R 1513 \\
F R 1514
\end{array}\right\}=\left[\begin{array}{llll}
X & O & O & O \\
O & X & O & O \\
O & O & X & O \\
X & O & O & X
\end{array}\right]\left\{\begin{array}{l}
D P 1511 \\
D P 1512 \\
D P 1513 \\
D P 1514
\end{array}\right\} \ldots(7)
$$

The conditions imposed to meet the FRs are incorporated in the synthesis technique. The six-bar Watt-I linkage is a single degree of freedom system and we can attain relative Z-motion (FR 1511). To avoid camber and caster changes (FR 1513), we specify that the motion of the two steering axis joints $A$ and $B$ be the same (All $\boldsymbol{\delta}_{j}$ are the same for both joints as shown in Figure 7). To avoid tire-wear (FR 1512), we specify the imaginary parts of all $\boldsymbol{\delta}_{j}$ to be zero. This ensures the wheel moves straight up and down, and not sideways, ensuring $\Delta \mathrm{y}=0$. FR 1514 can be easily met by specifying $a_{j}$

Figure 7 shows four prescribed positions of the two joints of the steering axis. We formulate the standard-form equations for the three dyads for the three desired displacements $\boldsymbol{\delta}_{1}, \boldsymbol{\delta}_{2}$ and $\boldsymbol{\delta}_{3}$. This gives us the 9 equations given below. Each of these 9 complex equations actually consists of two equations- one equating the real parts and the other equating the imaginary parts of the complex equation. Note that, since $\mathbf{Z}_{2}$ and $\mathbf{Z}_{4}$ are a part of the same rigid body, they undergo the same angular displacements $\beta_{i}$. Same is the case for $\mathbf{Z}_{3}$ and $\mathbf{Z}_{5}$, which have equal angular displacements $Y_{i}$

For the first dyad $\left(\mathbf{Z}_{1}\right.$ and $\left.\mathbf{Z}_{2}\right)$

$$
\begin{aligned}
& \boldsymbol{\delta}_{1}=\mathbf{Z}_{1}\left(e^{i \boldsymbol{\alpha}_{1}}-1\right)+\mathbf{Z}_{2}\left(e^{i \boldsymbol{\beta}_{1}}-1\right) \\
& \boldsymbol{\delta}_{2}=\mathbf{Z}_{1}\left(e^{i \boldsymbol{\alpha}_{2}}-1\right)+\mathbf{Z}_{2}\left(e^{i \boldsymbol{\beta}_{2}}-1\right) \cdots \\
& \boldsymbol{\delta}_{3}=\mathbf{Z}_{1}\left(e^{i \boldsymbol{\alpha}_{3}}-1\right)+\mathbf{Z}_{2}\left(e^{i \boldsymbol{\beta}_{3}}-1\right)
\end{aligned}
$$

For the second dyad $\left(\mathbf{Z}_{3}\right.$ and $\left.\mathbf{Z}_{4}\right)$

$$
\begin{aligned}
& \boldsymbol{\delta}_{1}=\mathbf{Z}_{3}\left(e^{i \boldsymbol{\gamma}_{1}}-1\right)+\mathbf{Z}_{4}\left(e^{i \boldsymbol{\beta}_{1}}-1\right) \\
& \boldsymbol{\delta}_{2}=\mathbf{Z}_{3}\left(e^{i \boldsymbol{\gamma}_{2}}-1\right)+\mathbf{Z}_{4}\left(e^{i \boldsymbol{\beta}_{2}}-1\right) \cdots \\
& \boldsymbol{\delta}_{3}=\mathbf{Z}_{3}\left(e^{i \boldsymbol{\gamma}_{3}}-1\right)+\mathbf{Z}_{4}\left(e^{i \boldsymbol{\beta}_{3}}-1\right)
\end{aligned}
$$

For the third dyad $\left(\mathbf{Z}_{5}\right.$ and $\left.\mathbf{Z}_{6}\right)$

$$
\begin{aligned}
& \boldsymbol{\delta}_{1}=\mathbf{Z}_{5}\left(e^{i \boldsymbol{\gamma}_{1}}-1\right)+\mathbf{Z}_{6}\left(e^{i \boldsymbol{\theta}_{1}}-1\right) \\
& \boldsymbol{\delta}_{2}=\mathbf{Z}_{5}\left(e^{i \boldsymbol{\gamma}_{2}}-1\right)+\mathbf{Z}_{6}\left(e^{i \boldsymbol{\theta}_{2}}-1\right) \cdots \\
& \boldsymbol{\delta}_{3}=\mathbf{Z}_{5}\left(e^{i \boldsymbol{\gamma}_{3}}-1\right)+\mathbf{Z}_{6}\left(e^{i \boldsymbol{\theta}_{3}}-1\right)
\end{aligned}
$$

The values of $\boldsymbol{\delta}_{1}, \boldsymbol{\delta}_{2}$ and $\boldsymbol{\delta}_{3}$ depend on the desired suspension travel (FR 1511). We can specify $\alpha_{1}, \alpha_{2}$, and $a_{3}$ based on the desired hardening characteristics. $\delta_{3} / a_{3}$ $>\delta_{2} / a_{2}$ will give a hardening suspension (FR 1514).

The first set of equations has 6 equations and 7 unknowns, $\mathbf{Z}_{1}, \mathbf{Z}_{2}, \beta_{1}, \beta_{2}$ and $\beta_{3}$. $\mathbf{Z}_{1}$ and $\mathbf{Z}_{2}$ are planar vectors and hence constitute four unknowns. We can fix one unknown and solve for the other six. This gives us $\mathbf{Z}_{1}$ and $\mathbf{Z}_{2}$.
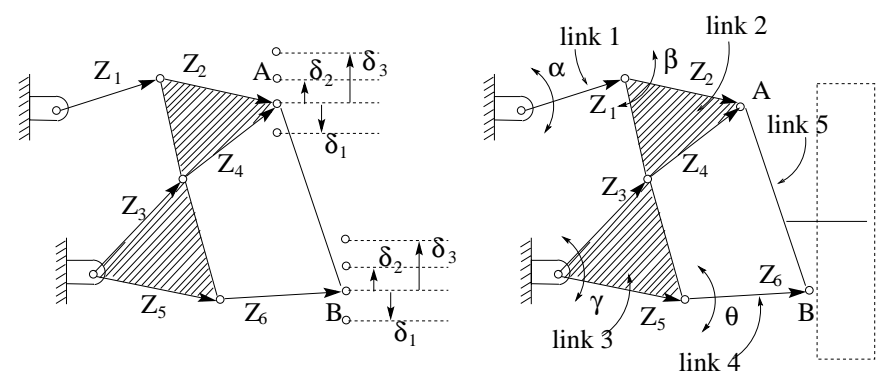

\section{Figure 7 : Dimensional synthesis for the six-bar Watt-I linkage}

The second set of equations has $\beta_{1}, \beta_{2}$ and $\beta_{3}$ specified from the previous equation. This again is a set of 6 equations with 7 unknowns. We can fix one unknown and solve for the other six to get $\mathbf{Z}_{3}$ and $\mathbf{Z}_{4}$. Similarly, we can solve for $\mathbf{Z}_{5}$ and $\mathbf{Z}_{6}$ from the third set of equations. The other link lengths can be obtained from vector additions of the known vectors $\mathbf{Z}_{1}$ through $\mathbf{Z}_{6}$.

\section{PROPOSED DESIGN OF STEERING SYSTEM}

\section{Existing steering system}

Figure 8 shows the top view schematic of the existing steering systems. Rotation of the steering wheel causes rotation of the Pitman arm through the steering column and the steering gear. This motion of the Pitman arm is transmitted through the tie rod to the steering knuckle which is rigidly connected to the vehicle wheel. This linkage transmits the motion of the steering wheel to the vehicle wheel. The two joints of the tie-rod are ball and socket joints to allow the suspension travel (in this case in and out of the plane of the paper).

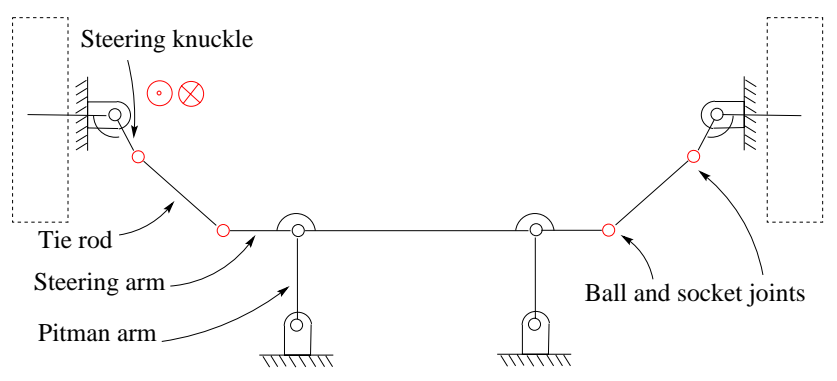

\section{Figure 8: Top view schematic of existing steering system}

\section{Need for a new steering system}

As discussed in a previous section, toe-changes due to suspension travel in existing suspension systems could cause tire-wear, directional instability, bump steer and the nibble problem. Also suspension travel changes the effective tie-rod length according to the relation 
$\mathrm{L}=\mathrm{L}_{1} \cos \theta$. The steering mechanism link lengths are designed for, amongst other features, toe-out on turns. Thus the toe-out on turns characteristics of the linkage will change under overload load causing unnecessary tire-wear

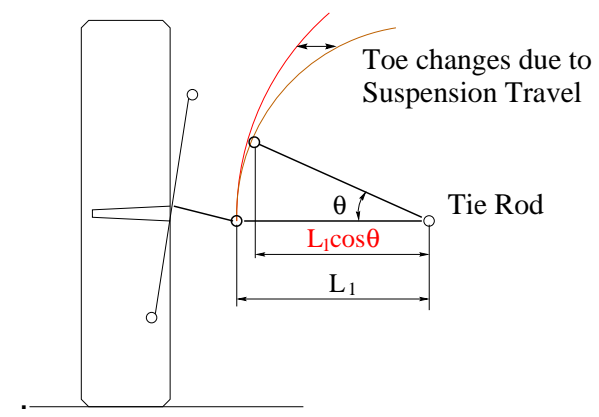

Figure 9 : Suspension travel causes toe-change and change in turning characteristics

Also the existing steering system is incompatible with the new six-bar suspension system as it shows excessive toe-sensitivity as shown in Figure 10 due to the vertical suspension travel.

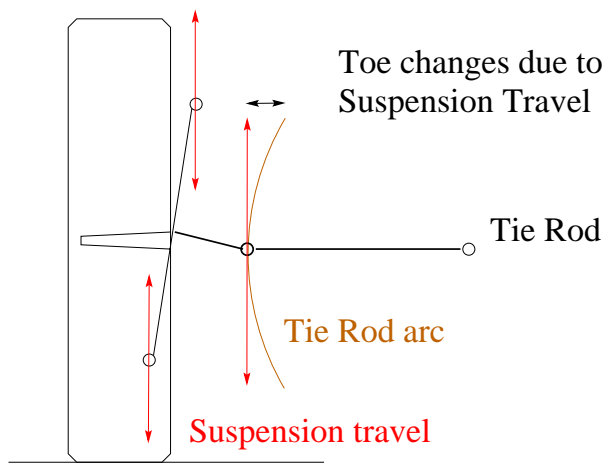

Figure 10 : Existing steering system is incompatible with the proposed suspension systems due to excessive toe-sensitivity

\section{Design of a new steering system}

Figure 11 shows the top view schematic of the proposed steering system. The proposed modification has a tie rod with a revolute joint at the inner tie rod end and a cylindrical joint at the outer tie rod end, instead of the ball and socket joints at both tie rod ends. In the new six-bar suspension system, the steering knuckle moves exactly vertically (in the Z-direction). It does not have any horizontal motion or any angle changes. This allows the use a cylindrical joint in place of ball and socket joints.

This system has a drawback that it does not allow the camber to change. We desire a camber roll on turns for suitable turning characteristics [3]. Hence we need to modify this steering system to allow camber roll on turns.

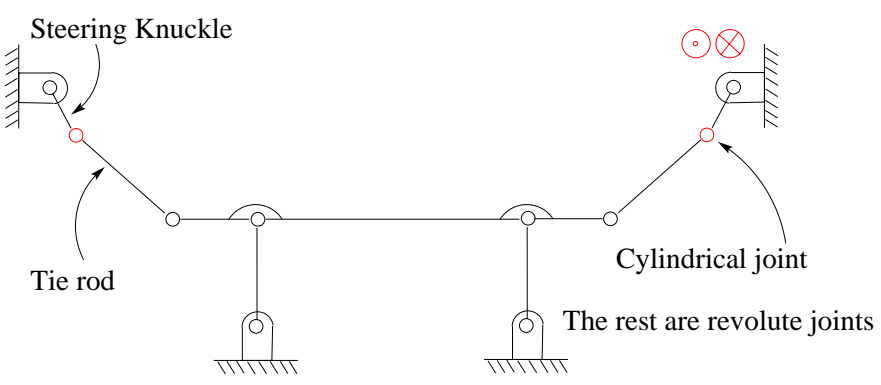

Figure 11 : Top view schematic of the proposed steering system

Modifications to allow camber change

To allow camber changes, a universal joint is introduced between the outer tie-rod end and the cylindrical joint as shown in Figure 12. Thus the steering system does not restrict camber changes and hence allows for camber roll on turns possible. Note that although the steering system does not restrict the camber changes, the suspension system does.

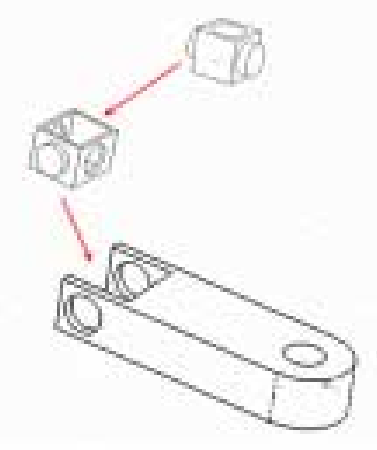

Figure 12 : Modification for camber roll on turns EVALUATION OF THE PROPOSED DESIGN

\section{ADVANTAGES} Wheel Alignment Parameters: Independent of
suspension travel

Equations 5 and 11 show the design matrices for the existing and proposed suspension and steering systems. The elements $X_{1}, X_{2}$ and $X_{3}$ in equation 5 indicate the coupling in the existing design. The DM for the proposed design is a lower triangular matrix and hence satisfies the Independence axiom. Moreover, the design is uncoupled with respect to the dynamic DPs, namely DP 152 (Suspension travel) and DP 153 (Wheel angle), i.e. these DPs do not affect any other FR.

A careful look at the DM for the existing systems indicates that we must fix DP 152 before DP 1422 and DP 1423. But this is a contradiction since DP 1422 is static in most cases and DP 1423 is static in all cases, whereas DP 152 is dynamic. 


$$
\left\{\begin{array}{c}
F R 151 \\
F R 1421 \\
F R 1422 \\
F R 1423 \\
F R 152 \\
F R 153
\end{array}\right\}=\left[\begin{array}{llllll}
X & O & O & O & O & O \\
X & X & O & O & O & O \\
X & X & X & O & O & O \\
X & X & O & X & O & O \\
X & O & O & O & X & O \\
X & O & O & O & O & X
\end{array}\right]\left\{\begin{array}{c}
D P 151 \\
D P 1421 \\
D P 1422 \\
D P 1423 \\
D P 152 \\
D P 153
\end{array}\right\} \cdots
$$

Both the DMs show that DP 1422 (Damping coefficient) does not affect any FR other that FR 1422. Axiomatic design theory indicates that DP 1422 can be used as a dynamic DP. It is indeed used in variable damping suspension systems and slow-active suspension systems. This is another example of application of axiomatic design theory to facilitate the rapid identification of such novel ideas through the FR/DP decomposition and the DM.

In the proposed system, WAP are independent of suspension travel and hence there are no unwanted turning torque changes $\left(X_{1}=0, X_{2}=0\right)$. This leads to better control. Since the WAP are independent of suspension, the problems of directional instability and tire-wear due to overload, offset load or road undulations are also eliminated. Since $X_{3}=0$ and $X_{4}=0$ in the proposed design, spring stiffness does not affect WAP or turning torque. Hence spring stiffness can be designed based only on comfort considerations and independent of control consideration. This will lead to better comfort.

\section{$\underline{\text { Hardening characteristics }}$}

We want suspension to be responsive to the bumps for small displacements, so that we get a smooth ride. But we also want to limit the relative displacements between the wheel and the frame to some reasonable value. This can be achieved if we have a suspension that hardens with displacement.

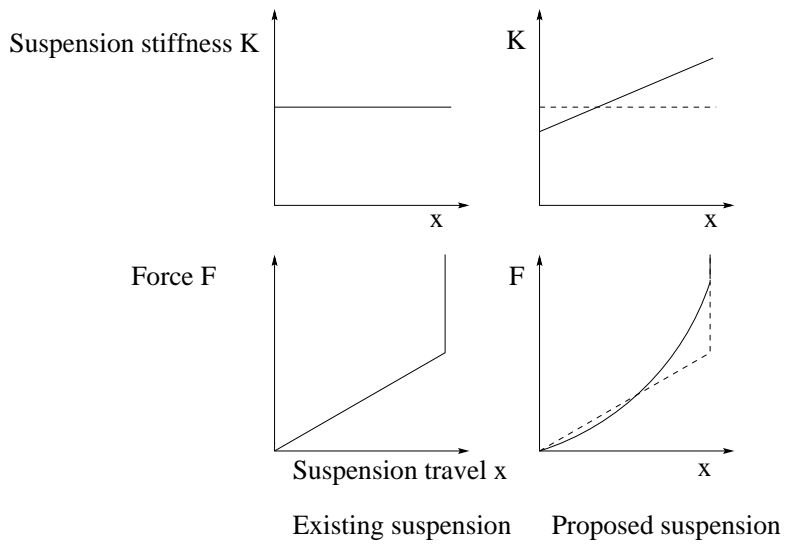

\section{Figure 13 : Hardening characteristics in proposed} suspension

In existing suspension systems, the spring compression or extension is directly proportional to suspension travel, giving a linear relation between force and suspension travel as shown in Figure 13. The force increases suddenly when the control arm hits the jounce bumper.

In the proposed six-bar Watt-I linkage, suitable design can achieve a non-linear relation between the suspension travel and spring compression giving rise to a progressive effective spring rate and a hardening suspension as shown in Figure 13.

\section{LIMITATIONS}

The six-bar Watt-I linkage has the disadvantages of more number of links and joints, higher cost and higher unsprung weight.

\section{Unfavorable camber changes due to body roll and possible solutions}

The proposed suspension maintains $\Delta \varnothing=0$, but $\varnothing$ is measured with respect to the vehicle frame, whereas camber is measured with respect to ground. If the vehicle frame tilts with respect to the ground (exhibits roll), we will have an equal and opposite camber on both wheels, with magnitude equal to the vehicle roll. This leads to a positive camber on the outer wheels, which is unfavorable on turns.

One solution to avoid this problem could be to eliminate body-roll. This can be done by active roll-stabilization (ARS). Several existing vehicles have this ARS feature. Incorporating ARS will further improve handling and comfort levels.

\section{CONCLUSION}

This paper presents an axiomatic design approach to remove the coupling in the vehicle suspension and steering systems. FR/DP decomposition of the existing suspension and steering system is presented and coupling is identified. A new suspension system has been proposed which removes the coupling by making the wheel alignment parameters independent of suspension travel and hence delivers a better performance in terms of comfort, control and tire-wear. An analytical technique for the kinematic synthesis of the suspension system using a six-bar Watt-I mechanism is presented. A new steering system conformal to the new suspension system has been proposed. FR/DP decomposition of the vehicle system is presented. This indicates other couplings and DP redundancies in the vehicle system and also provides a framework for design of novel vehicles.

\section{ACKNOWLEDGMENTS}

This research was carried out at MIT and was made possible by the funding from Ford-MIT Alliance. The authors would like to thank Dr. Tim Davis of Ford Motor Company and Steven Schondorf, Kristin Schondorf and Prof. John Heywood of the Ford-MIT alliance for their support. The authors would also like to thank Steve 
Allen, Ben Maher and Jason Sterly of Ford Motor Company for their help.

\section{REFERENCES}

1. N P Suh. Axiomatic Design: Advances and Applications.CIRP Design Book Series. Oxford University Press, 2001.

2. N P Suh. The Principles of Design. Oxford University Press, New York, 1990.

3. J Reimpell, H Stoll and J W Betzler. The Automotive Chassis. Society of Automotive Engineers, Warrendale, PA. 1996

4. J W Melvin and N P Suh. Beyond the Hierarchy: System-wide rearrangement as a tool to eliminate iteration. Proceedings of the International Conference on Axiomatic Design. Cambridge, MA, 2002.

5. Donald Bastow. Car Suspension and Handling. Pentech, London, 1987.

6. G N Sandor and A G Erdman. Advanced Mechanism Design: Analysis and Synthesis, volume 2. PrenticeHall, New Jersey, 1989.

7. W F Milliken and D L Milliken. Race Car Vehicle Dynamics. Society of Automotive Engineers, Warrendale, PA, 1995.
8. T D Gillespie. Fundamentals of Vehicle Dynamics. Society of Automotive Engineers, Warrendale, PA, 1992.

\section{CONTACT}

Hrishikesh Deo, S.M.(MIT,2003) B.Tech (IIT Bombay, 2001), is pursuing doctoral studies at the Department of Mechanical Engineering, Massachusetts Institute of Technology.<hvd@mit.edu>

Nam $P$ Suh, is the Ralph E. and Eloise F. Cross Professor, Department of Mechanical Engineering, MIT. $\mathrm{He}$ has made exceptional contributions in the fields of tribology, materials processing, manufacturing, design theory and engineering education. He has published over 250 papers and 8 books, and holds over 50 US patents. <npsuh@mit.edu>

\section{DEFINITIONS, ACRONYMS, ABBREVIATIONS}

WAP: Wheel Alignment Parameters

FR: Functional Requirement

DP: Design Parameter

DM: Design Matrix 